\title{
A novel multiscale model for the simulation of polymer flash nano-precipitation
}

DOI:

10.1016/j.ces.2017.04.047

\section{Document Version}

Accepted author manuscript

Link to publication record in Manchester Research Explorer

\section{Citation for published version (APA):}

Lavino, A. D., Di Pasquale, N., Carbone, P., \& Marchisio, D. (2017). A novel multiscale model for the simulation of polymer flash nano-precipitation. Chemical Engineering Science, 171. https://doi.org/10.1016/j.ces.2017.04.047

\section{Published in:}

Chemical Engineering Science

\section{Citing this paper}

Please note that where the full-text provided on Manchester Research Explorer is the Author Accepted Manuscript or Proof version this may differ from the final Published version. If citing, it is advised that you check and use the publisher's definitive version.

\section{General rights}

Copyright and moral rights for the publications made accessible in the Research Explorer are retained by the authors and/or other copyright owners and it is a condition of accessing publications that users recognise and abide by the legal requirements associated with these rights.

\section{Takedown policy}

If you believe that this document breaches copyright please refer to the University of Manchester's Takedown Procedures [http://man.ac.uk/04Y6Bo] or contact uml.scholarlycommunications@manchester.ac.uk providing relevant details, so we can investigate your claim.

\section{OPEN ACCESS}




\title{
A novel multiscale model for the simulation of polymer flash nano-precipitation
}

\author{
Alessio D. Lavino ${ }^{\mathrm{a}}$, Nicodemo di Pasquale ${ }^{\mathrm{b}}$, Paola Carbone ${ }^{\mathrm{c}}$, Daniele L. \\ Marchisio $^{\mathrm{a}, *}$ \\ ${ }^{a}$ Department of Applied Science and Technology, Institute of Chemical Engineering, \\ Politecnico di Torino, 10129 Torino, Italy \\ ${ }^{b}$ School of Chemistry, The University of Manchester, Oxford Road, Manchester M13 \\ 9PL, UnitedKingdom \\ ${ }^{c}$ School of Chemical Engineering and Analytical Science, The University of Manchester, \\ Oxford Road, Manchester M13 9PL, UnitedKingdom
}

\begin{abstract}
Numerous models describe the flash nano-precipitation (FNP) process to form polymer nanoparticles, however most of them are based on equilibrium approaches and are not capable of predicting kinetically stable configurations. Moreover, since FNP occurs through solvent-displacement, the way in which the solvent and anti-solvent are mixed plays an important role, which is often overlooked. Here we propose a multiscale approach, which combines molecular dynamics (MD), a Smoluchowski population balance equation (PBE) and computational fluid dynamics (CFD), to model the FNP process, from the atomistic-scale up to the macro-scale. The particle formation process is not described with the usual nucleation-and-growth approach, but as Brownian aggregation of the polymer molecules into nanoparticles. Being the final nanoparticles amorphous, no energy barrier to the aggregation process is considered, whereas the effects of both turbulent mixing and turbulent aggregation on the evolution of the nanoparticles are accounted for. The main novelty of this work is that the aggregation kernel appearing in the PBE,
\end{abstract}

\footnotetext{
*Corresponding author: Daniele Marchisio; e-mail: daniele.marchisio@polito.it; tel.: +390110904622; fax: +390110904699

Email addresses: alessio.lavino@polito.it (Alessio D. Lavino), nicodemo.dipasquale@manchester.ac.uk (Nicodemo di Pasquale), paola.carbone@manchester.ac.uk (Paola Carbone), daniele.marchisio@polito.it (Daniele L. Marchisio)
} 
coupled in turn with CFD, is calculated from MD simulations, following the multiscale modeling paradigm. The model is tested on the FNP of poly- $\varepsilon-$ caprolactone nanoparticles in acetone-water mixtures. Predictions for the final mean nanoparticle size are found in good agreement with experiments, especially at high initial polymer concentrations, where the hypothesis of no energy barrier is more realistic.

Keywords: Multiscale modeling, QMOM, Population balance equation, Computational fluid dynamics, Solvent-displacement, flash-nano precipitation

\section{Introduction}

Although the flash nano-precipitation (FNP) process of polymer nanoparticles has been extensively investigated, both with experimental and modeling approaches, numerous are the challenges and the issues, that still need to be addressed. Above all, this kind of systems is simulated by using models, designed to describe what happens at the equilibrium (Jones, 2002). These neglect therefore kinetic effects, which are also well-known to play an important role (Johnson and Prud'homme, 2003b; Lince et al., 2008), in determin-

ing, for example, the size and structure of the final nanoparticles (Celasco et al., 2014; Valente et al., 2012a b). Another important limitation of the developed models is that the nanoparticle formation process is investigated and modeled only at one scale, by using for example molecular dynamics (MD), Monte Carlo (MC) methods and various coarse-grained models (Sun and Yang, 2014; Wang and Larson, 2015; Yuan et al., 2010). While these methods can capture the molecular interactions, they are unable to account for the effect that the fluid flow has on the nanoparticle formation process, particularly important when investigating the scale-up of these processes or their transfer from batch to continuous, as it happens in continuous manufacturing. Moreover they are inherently constrained at time- and length-scales which are too small to model system size comparable to the experimental ones Capretto et al., 2011; Li et al., 2006; Rajagopalan, 2001; Yan and Xie, 2013).

We want to overcome these limitations by developing a novel multiscale model, that accounts for kinetic effects and that addresses both the molecular-scale, as well as macro-scale. The model is here validated for the case of FNP of poly- $\varepsilon$-caprolactone nanoparticles in acetone-water mixtures. 
PCL is a saturated aliphatic polyester with hexanoate repeated units, employed in a wide range of applications, ranging from biomedical devices to controlled drug-delivery systems. Its popularity is due to its biocompatibility with the human body (Who et al., 2000), as well as to the harmlessness of the degradation products (Maeda, 2001). The methodology developed and tested in this work has however a wider range of application, as it can be used also to describe other "soft systems" such as surfactants, colloids, liquid crystals, proteins, DNA (Bockstaller et al., 2005; Cohen et al., 2011a b c d; Collins et al., 2004; Ferrone et al., 1985; Knowles et al., 2009; Oosawa and Asakura, 1975; Oosawa and Kasai, 1962; Zhou and Ferrone, 1990). Moreover, it could also be used as an exploratory tool to estimate the effect of fluid dynamics on nucleation, following recent works on this topic (Mura and Zaccone, 2016).

The experimental technique used to induce the FNP is the solvent-displacement method, where the PCL polymer molecules are dissolved in a so-called "good" solvent (e.g. acetone) and the solution is then mixed with a "bad" solvent or anti-solvent (e.g. water) for the polymer, but with which the "good" solvent is fully miscible. The presence of the poor solvent induces the polymer molecules aggregation in clusters or nanoparticles of variable sizes (Jones, 2002). Solvent-displacement often takes place in very small continuous mixers, such as the confined impinging jets mixer (Johnson and Prud'homme, 2003a) (CIJM), where solvent and anti-solvent are mixed together continuously and in a controlled way (Johnson and Prud'homme, 2003b), completely defining the environment in which FNP occurs.

The multiscale model developed in this work describes the FNP as an aggregation process, as also proposed by other authors (Cheng et al., 2010), but for the first time it calculates the rate with which polymer molecules interact (via the so-called aggregation kernel) directly from MD simulations (Di Pasquale et al., 2014). MD data are, in fact, used to fit the Flory law (Flory, 1953; Rubinstein and Colby, 2003) for the radius of gyration and the Stokes-Einstein law for the diffusion coefficient of single PCL polymer molecules, completely defining the aggregation kernel appearing in the Smoluchowski population balance equation (PBE). This fitting is then extended to clusters of polymer molecules to predict the evolution of the cluster mass distribution (CMD), in turn employed to quantify the polydispersity of the nanoparticles in terms of the number of polymer molecules belonging to a polymer nanoparticle. The evolution of the CMD is dictated by the PBE, solved with the quadrature method of moments (QMOM) (Marchisio et al., 
2003) inside a computational fluid dynamics (CFD) code, to account for the inhomogeneous mixing between the acetone and water streams and for the influence of turbulence on the process. Including mixing in the description of FNP is particularly important, as FNP is very fast, almost instantaneous, mixing-limited (Cheng et al., 2010) and irreversible, and is therefore strongly influenced by spatial inhomogeneities (Johnson and Prud'homme, 2003a b). Being, for the test cases considered in this work, the flow turbulent, it is also necessary to consider the effect of turbulent fluctuations on the aggregation process. This is done, in the Favre-averaged Navier-Stokes equations (FANS) framework by using the direct quadrature method of moments coupled with the interaction and exchange with the mean (DQMOM-IEM) model (Fox, 2003). As it clearly emerges the FNP process is here described on three different scales: molecular-scale, by using MD, cluster-scale, by means of the Smoluchowski PBE, and macro-scale, by means of CFD.

The model is in this work validated against experimental data referring to different mixing conditions in a CIJM, different initial polymer concentrations and two different molecular weights for the PCL. Comparison with experimental data allows to assess the range of validity of the approach, in line with the theory, and to conclude that this multiscale approach not only provides a molecular insight into the structure of the polymer nanoparticles but also seems to be more effective than other alternatives, based for example on the classical nucleation theory (CNT) (Di Pasquale et al., 2012; Garcia et al., 2014) or other more detailed, but similar, theories in predicting macroscopic data (Di Pasquale et al., 2013).

\section{Governing equations}

As already mentioned turbulent mixing between acetone and water is described here with CFD. Turbulence is treated with the FANS approach, since two fluids with different densities are involved. The continuity equation for the acetone-water mixture is solved together with the Navier-Stokes equations, the equations for the turbulent kinetic energy, $k$, and the turbulent dissipation rate, $\varepsilon$, as common practice with the standard $k-\varepsilon$ model. As these equations are very well known and are already implemented in the CFD code, they are omitted here and readers interested in the details can refer, for example, to our previous work (Di Pasquale et al., 2012). As the main novelty of this work consists in the use of the PBE, a detailed summary of the equations employed and implemented in the CFD code follows. 
In order to efficiently describe the evolution of the population of polymer molecule clusters, a Smoluchowski PBE is employed. In what follows we will refer to the polymer particles as nanoparticles or molecular clusters, interchangably. The PBE operates on the CMD, $f(\mathbf{x}, n)$, defined in such a way that the quantity, $f(\mathbf{x}, n) \mathrm{d} n$, represents the number density of molecular clusters or nanoparticles containing $n$ polymer molecules at position $\mathbf{x}$. The variable $n$, referred to in what follows as dimensionless cluster mass, is discrete, but since it varies between one and very large numbers, is treated here as a continuous variable. It is also useful to highlight here that, in the acetone inlet, when the polymer molecules are dissolved in the solvent, and do not show any tendency to self-assemble, the CMD is always equal to zero, except for $n=1$, where it assumes the value corresponding to the initial polymer molecule number density in acetone. Moreover, as the values assumed by the CMD would inevitably be very large, the CMD is normalized by dividing it with the Avogadro number. By using this normalization the CMD in the acetone inlet corresponds to the initial polymer molar concentration.

The evolution of the CMD is defined by the PBE, that for a stationary problem and before Favre average, reads as follows (Marchisio and Fox, 2013):

$$
\begin{aligned}
& \frac{\partial}{\partial x_{i}}\left(U_{i} f(n)\right)-\frac{\partial}{\partial x_{i}}\left(D(n) \frac{\partial f(n)}{\partial x_{i}}\right)= \\
& \quad=\frac{1}{2} \int_{0}^{n} \beta\left(n-n^{\prime}, n^{\prime}\right) f\left(n-n^{\prime}\right) f\left(n^{\prime}\right) \mathrm{d} n^{\prime}-\int_{0}^{\infty} \beta\left(n, n^{\prime}\right) f(n) f\left(n^{\prime}\right) \mathrm{d} n^{\prime},
\end{aligned}
$$

where the Einstein notation was used and space dependencies were omitted for brevity, $D(n)$ is the diffusion coefficient due to Brownian motions of a nanoparticle of size $n, U_{i}$ is the nanoparticle velocity and $\beta\left(n, n^{\prime}\right)$ is the aggregation kernel. It is assumed here that $U_{i}$ is identical to the fluid velocity, as nanoparticles are very small and characterized by a density very similar to that of the acetone-water mixture (Baldyga and Orciuch, 2001) (i.e. pseudo-homogeneous system hypothesis). The aggregation kernel quantifies the rate with which polymer molecule clusters (or nanoparticles) of different size aggregate together forming a larger cluster and its functional form will be introduced later on. It is important to stress here that aggregation results in two source terms, one positive related to the formation of a cluster of size $n$ and one negative due to the disappearance of clusters of size $n$. Since the solution of the PBE with discretized methods (Marchisio and Fox, 2013) leads to very high computational costs, it is more convenient to solve the 
problem in terms of the moments of the CMD. The moment of order $j$ is defined as follows:

$$
m^{(j)}=\int_{0}^{\infty} f(n) n^{j} \mathrm{~d} n .
$$

An additional advantage of solving the PBE in terms of the moments of the CMD is that they represent physical measurable quantities; in fact, $m^{(0)}$ represents the total nanoparticle number density, $m^{(1)}$ is a conserved quantity and represents the total number density of polymer molecules, whereas the ratio between $m^{(1)}$ and $m^{(0)}$ results in the average number of polymer molecules per nanoparticle. Furthermore, by using the first three order moments, it is possible to build up the CMD, through suitable algorithms (Fox, 2003). By applying the moment transform the steady-state transport equation for the moment of order $j$ assumes the following form:

$$
\begin{aligned}
& \frac{\partial}{\partial x_{i}}\left(\left\langle U_{i}\right\rangle\langle m\rangle^{(j)}\right)-\frac{\partial}{\partial x_{i}}\left(D_{t} \frac{\partial\langle m\rangle^{(j)}}{\partial x_{i}}\right)= \\
& =\frac{1}{2} \iint_{0}^{\infty}\left\langle\left[\left(n+n^{\prime}\right)^{j}-n^{j}-n^{\prime j}\right] \beta\left(n, n^{\prime}\right) f(n) f\left(n^{\prime}\right) \mathrm{d} n \mathrm{~d} n^{\prime}\right\rangle,
\end{aligned}
$$

where $\left\langle U_{i}\right\rangle$ and $\langle m\rangle^{(j)}$ are the Fauvre-averaged velocity and moment of the CMD, respectively, and where during derivation it was assumed that the turbulent diffusivity, $D_{t} \approx \mu_{t} / 0.7 \rho$, which does not depend on the moment order, is much larger than the Brownian diffusivity and is calculated from the turbulent viscosity, $\mu_{t}$, by assuming a turbulent Schmidt number of 0.7 (Andersson et al., 2012). The integrals that appear on the right-hand side of Eq. (3) are affected by a closure problem due to the impossibility of writing the integrals in terms of the moments of the CMD. To overcome this closure problem QMOM is used, with four moments (i.e. $j=0, \ldots, 3$ ), corresponding to a quadrature approximation with two nodes. When the Fauvre average is also applied a second closure problem arises due to the fact that turbulent fluctuations, that affect the CMD, are characterized by time-scales comparable with that of aggregation. To overcome this second closure problem DQMOM-IEM, with two nodes or environments, is employed. Details concerning these methods can be found elsewhere $(\overline{F o x}, 2003$; Marchisio and Fox, 2013).

Let us now define the functional form of the aggregation kernel, $\beta\left(n, n^{\prime}\right)$, predicting the rate of aggregation of clusters of size $n$ with clusters of size $n^{\prime}$, 
appearing in Eq. (3). To do that it is useful to know that our MD simulations, performed in a previous work (Di Pasquale et al., 2012), have shown that the PCL molecule behaves as a freely-jointed chain (Rubinstein and Colby, 2003). This observation allows to assume that the behavior of a molecular cluster or nanoparticle with $n$ PCL molecules of molecular weight $M_{w}$, is similar to the behavior of a single PCL molecule with molecular weight $n M_{w}$. This assumption allows to extend the Flory law, valid for a single PCL molecule:

$$
\llbracket R_{g}^{2} \rrbracket=k M_{w}^{2 \nu},
$$

to a cluster of $n$ PCL molecules:

$$
\llbracket R_{g}^{2}(n) \rrbracket=k\left(n M_{w}\right)^{2 \nu},
$$

where $\llbracket R_{g}{ }^{2} \rrbracket=\llbracket R_{g}{ }^{2}(n=1) \rrbracket$ is the ensemble-averaged squared radius of gyration of a single PCL molecule, $\llbracket R_{g}{ }^{2}(n) \rrbracket$ is the ensemble-averaged squared radius of gyration of a PCL cluster with $n$ molecules, $M_{w}$ is the molecular weight of a single PCL chain and $k$ and $\nu$ are the Flory parameters, that depend on the acetone molar fraction $x_{A}$ in the acetone-water mixture. Their functional dependences on $x_{A}$ were determined in our previous work via MD simulations (Di Pasquale et al., 2012) and are reported in the next section, namely in Eq. (10) and Eq. (11).

The aggregation kernel accounts for two mechanisms: collisions due to Brownian motions and due to turbulent fluctuations. By using the following approximation: $\llbracket R_{g} \rrbracket \simeq \sqrt{\llbracket R_{g}{ }^{2} \rrbracket}$ and by assuming that the diffusion coefficient due to Brownian motions can be calculated with the Stokes-Einstein equation (Di Pasquale et al., 2012), as supported by our MD simulations, the following expression is obtained (Cheng et al., 2010):

$$
\begin{gathered}
\beta\left(n, n^{\prime}\right)=\eta\left(\frac{2 k_{B} T}{3 \mu} \frac{\left(\llbracket R_{g}(n) \rrbracket+\llbracket R_{g}\left(n^{\prime}\right) \rrbracket\right)^{2}}{\llbracket R_{g}(n) \rrbracket \llbracket R_{g}\left(n^{\prime}\right) \rrbracket}+1.2944 \sqrt{\frac{\rho \varepsilon}{\mu}}\left(\llbracket R_{g}(n) \rrbracket+\llbracket R_{g}\left(n^{\prime}\right) \rrbracket\right)^{3}\right) \\
=\eta\left(\frac{2 k_{B} T}{3 \mu} \frac{\left(\sqrt{k\left(n M_{w}\right)^{2 \nu}}+\sqrt{k\left(n^{\prime} M_{w}\right)^{2 \nu}}\right)^{2}}{\sqrt{k\left(n M_{w}\right)^{2 \nu}} \sqrt{k\left(n^{\prime} M_{w}\right)^{2 \nu}}}+\right. \\
\left.+1.2944 \sqrt{\frac{\rho \varepsilon}{\mu}}\left(\sqrt{k\left(n M_{w}\right)^{2 \nu}}+\sqrt{k\left(n^{\prime} M_{w}\right)^{2 \nu}}\right)^{3}\right),
\end{gathered}
$$


where $\eta$ is the aggregation efficiency (more detailed on this in the next section), $\mu$ is the mixture dynamic viscosity, $k_{B}$ is the Boltzmann constant and $\varepsilon$ is the turbulent dissipation rate. Derivation of Eq. (6) also made use of the assumption that the two aggregation mechanisms are simply additive: at low PCL initial concentrations and low turbulence intensity the Brownian aggregation mechanism dominates on the turbulent one and viceversa at high PCL initial concentrations and high turbulence intensity.

\section{Simulation conditions and computational details}

In order to validate the model developed, predictions are compared with experiments obtained under different operating conditions. In the experiments the water and acetone streams are mixed in a CIJM continuously, as shown in Fig. 1. First of all, two molecular weights for PCL are simulated: the first one has $M_{w}=14000 \mathrm{~g} \mathrm{~mol}^{-1}$ (indicated in what follows as PCL-14000), whereas the second one has $M_{w}=80000 \mathrm{~g} \mathrm{~mol}^{-1}$ (indicated as PCL-80000). For both of them, different operating conditions have been considered, in terms of initial PCL concentrations (in the acetone stream) and inlet acetone and water flow rates, whereas the flow rate ratio between water and acetone was kept equal to one. Therefore, the final acetone concentration at the CIJM outlet in terms of volume, molar and mass fraction is respectively equal to $0.50,0.20$ and 0.44 .

The operating conditions related to PCL-14000 and PCL-80000 are summarized in Tab. 1. Among other numerous variables and parameters of interest, it is useful to discuss in depth the initial supersaturation ("I.S."), calculated as the ratio between the final PCL concentration (third column), after mixing of water and acetone streams, and the equilibrium PCL concentration, reported in Eq. (7). This value is very important since it represents the driving force for FNP. When it is smaller than one the PCL molecule concentration is smaller than its equilibrium value and therefore the solution is stable: PCL molecules collide but do not aggregate forming nanoparticles. In other words under these conditions the aggregation efficiency is null: $\eta=0$. On the contrary, when I.S. is much larger than one, PCL molecule aggregation is favored and upon collision molecules stick together forming a nanoparticle; under these conditions (i.e. I.S. $\gg 1$ ) it is plausible to assume that there is no resistance or barrier to the aggregation process and therefore the aggregation efficiency is equal to one: $\eta=1$. For moderate I.S. values a nucleative phenomenon might take place, corresponding to intermediate 


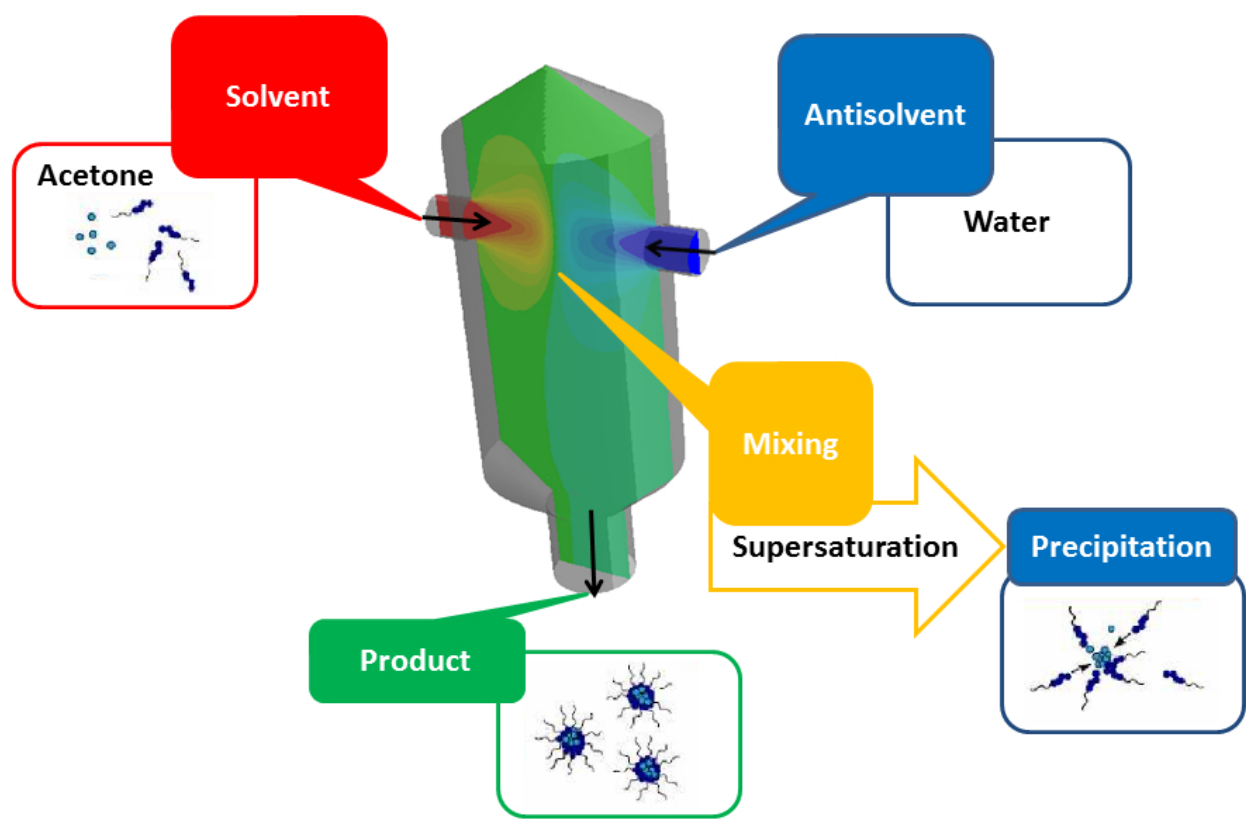

Figure 1: Sketch of the CIJM. Acetone, with PCL, and water flow respectively from left and right; black arrows show the flux lines. There may be also organic molecules (e.g. drug, light blue beads in the figure). The mixing leads to supersaturation and to the formation of polymer molecule clusters that may contain the organic molecules. In this work the presence of the latter is neglected.

aggregation efficiency values: $0<\eta<1$. Since however for the moment there is no simple way to determine the actual efficiency value under these conditions, it is assumed that $\eta=1$ also for intermediate I.S. values. The final comparison with experiments will allow us to draw some conclusions on this point.

The case referring to PCL with the smaller molecular weight (i.e. PCL14000) is first investigated under the effect of Brownian-induced aggregation only and then subsequently under the effect of both Brownian-induced and turbulent-induced aggregation, in order to assess the relative importance of the two mechanisms. For the other molecular weight (i.e. PCL-80000) only 
Table 1: Operating conditions adopted in the simulations related to PCL-14000 and to PCL-80000. The first and second columns refer to the initial PCL concentration in the acetone stream in $\mathrm{mg} \mathrm{mL}^{-1}$ and $\mathrm{mol} \mathrm{m} \mathrm{m}^{-3}$. The third column refers to the final PCL concentration in the outlet stream (after mixing of acetone and water streams). The fourth column quantifies the initial supersaturation (I.S.).

\begin{tabular}{|c|c|c|c|c|}
\hline$c_{\mathrm{in}}^{\mathrm{PCL}}$ & $c_{\mathrm{in}}^{\mathrm{PCL}}$ & $c_{\text {out }}^{\mathrm{PCL}}$ & I S & Flow rate $\left(\mathrm{mL} \min ^{-1}\right)$ \\
\hline$\left(\mathrm{mg} \mathrm{mL}^{-1}\right)$ & & & 1.S. & $\begin{array}{llllll}10 & 20 & 40 & 60 & 80 & 120\end{array}$ \\
\hline 0.50 & $3.57 \times 10^{-2}$ & $1.79 \times 10^{-2}$ & 23.30 & \multirow{6}{*}{ PCL-14000 } \\
\hline 2.50 & $1.79 \times 10^{-1}$ & $8.93 \times 10^{-2}$ & 116.70 & \\
\hline 5.00 & $3.57 \times 10^{-1}$ & $1.79 \times 10^{-1}$ & 233.40 & \\
\hline 10.0 & $7.14 \times 10^{-1}$ & $3.57 \times 10^{-1}$ & 466.70 & \\
\hline 15.0 & 1.07 & $5.36 \times 10^{-1}$ & 700.10 & \\
\hline 25.0 & 1.79 & $8.93 \times 10^{-1}$ & 1166.80 & \\
\hline 0.02 & $2.50 \times 10^{-4}$ & $1.25 \times 10^{-4}$ & 0.90 & \multirow{6}{*}{ PCL-80000 } \\
\hline 0.20 & $2.50 \times 10^{-3}$ & $1.25 \times 10^{-3}$ & 9.30 & \\
\hline 1.00 & $1.25 \times 10^{-2}$ & $6.25 \times 10^{-3}$ & 46.70 & \\
\hline 3.00 & $3.75 \times 10^{-2}$ & $1.86 \times 10^{-2}$ & 140.00 & \\
\hline 4.00 & $5.00 \times 10^{-2}$ & $2.50 \times 10^{-2}$ & 186.70 & \\
\hline 6.00 & $7.50 \times 10^{-2}$ & $3.75 \times 10^{-2}$ & 280.00 & \\
\hline
\end{tabular}

predictions accounting for both effects will be discussed.

As already mentioned FNP is induced by a change in the solvent. The aggregation efficiency, $\eta$, introduced in Eq. (6) is assumed to be zero if the PCL molecule concentration, $c^{\mathrm{PCL}}$, is smaller than the equilibrium concentration, $c_{\mathrm{eq}}^{\mathrm{PCL}}$, and equal to one otherwise. The equilibrium concentration is in other words the concentration at which PCL molecules start to aggregate into clusters/nanoparticles. The local PCL concentration is calculated from the local acetone mass fraction, $\xi$, (in turn function of the local acetone molar fraction $x_{A}$ ) via the following equation:

$$
c^{\mathrm{PCL}}=\frac{c_{\mathrm{in}}^{\mathrm{PCL}}}{M_{w}}\left[1+\left(\frac{1}{\xi}-1\right)\left(\frac{\rho_{A}}{\rho_{W}}\right)\right]^{-1},
$$

where $M_{w}$ is the molecular weight in $\mathrm{g} \mathrm{mol}^{-1}, c_{\mathrm{in}}^{\mathrm{PCL}}$ is the initial PCL concentration expressed in $\mathrm{mg} \mathrm{mL}^{-1}, \rho_{A}$ and $\rho_{W}$ are respectively the density of acetone and water; whereas the equilibrium concentration, $c_{\mathrm{eq}}^{\mathrm{PCL}}$, was determined experimentally in a previous work (Lince et al. 2008 ) and fitted in a second one (Di Pasquale et al., 2012) obtaining the exponential correlation reported in Fig. 2 and 3 for PCL-14000 and PCL-80000, respectively. 
The figures report the equilibrium condition in terms of the molar acetone fraction, $x_{A}$, as a function of the PCL equilibrium concentration together with the operating conditions investigated in the experiments. The symbols report the final PCL concentrations in the acetone-water mixture with the final solution composition, after complete mixing of the acetone and water solutions.

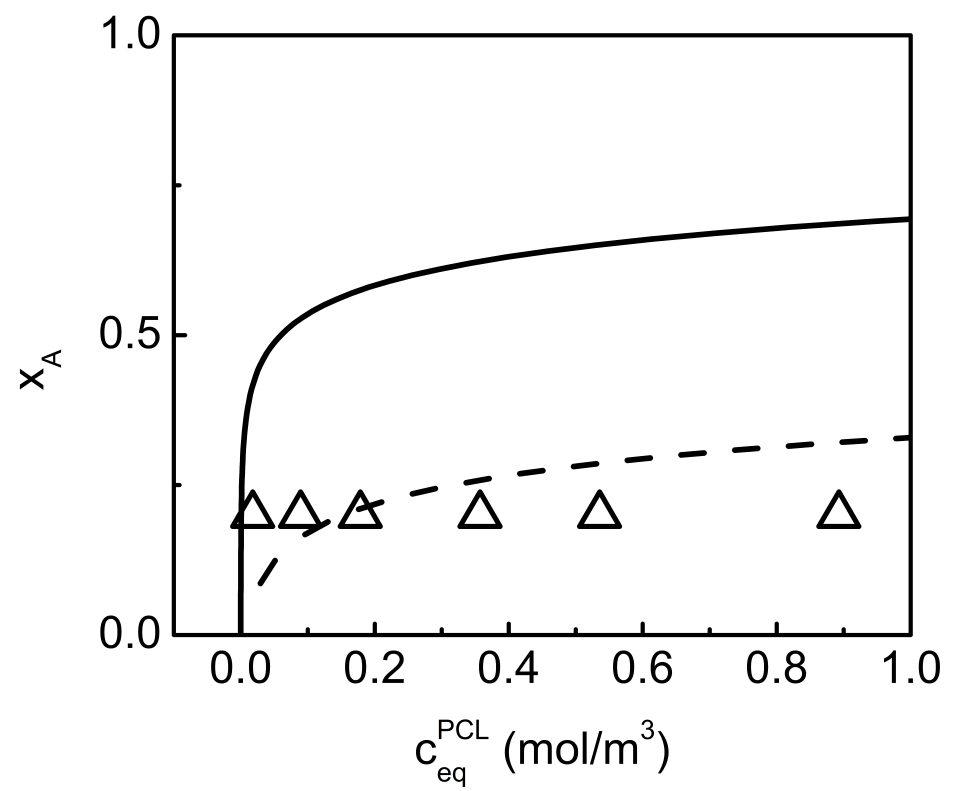

Figure 2: Solubility curve for PCL-14000 in acetone-water mixtures, reported as acetone molar fraction, $x_{A}$, versus the equilibrium concentration, $c_{\mathrm{eq}}^{\mathrm{PCL}}\left(x_{A}\right)$ (continuous line). Points above the curve correspond to stable mixture where PCL molecules do not aggregate, otherwise for the points below. The symbols (empty triangles) identify the polymer concentrations at the outlet, $c_{\text {out }}^{\mathrm{PCL}}$, for equal acetone and water flow rates, investigated in this work. The dashed curve corresponds to the supersaturation value equal to 200 . The reason for choosing this latter value will be explained later on.

The acetone-water mixture density is calculated from the following equation:

$$
\rho=\left(\frac{\xi}{\rho_{A}}+\frac{1-\xi}{\rho_{W}}\right)^{-1},
$$

where $\rho_{A}$ and $\rho_{W}$ are the acetone and water densities, respectively and, as already mentioned, $\xi$ is the acetone mass fraction in the mixture. The 


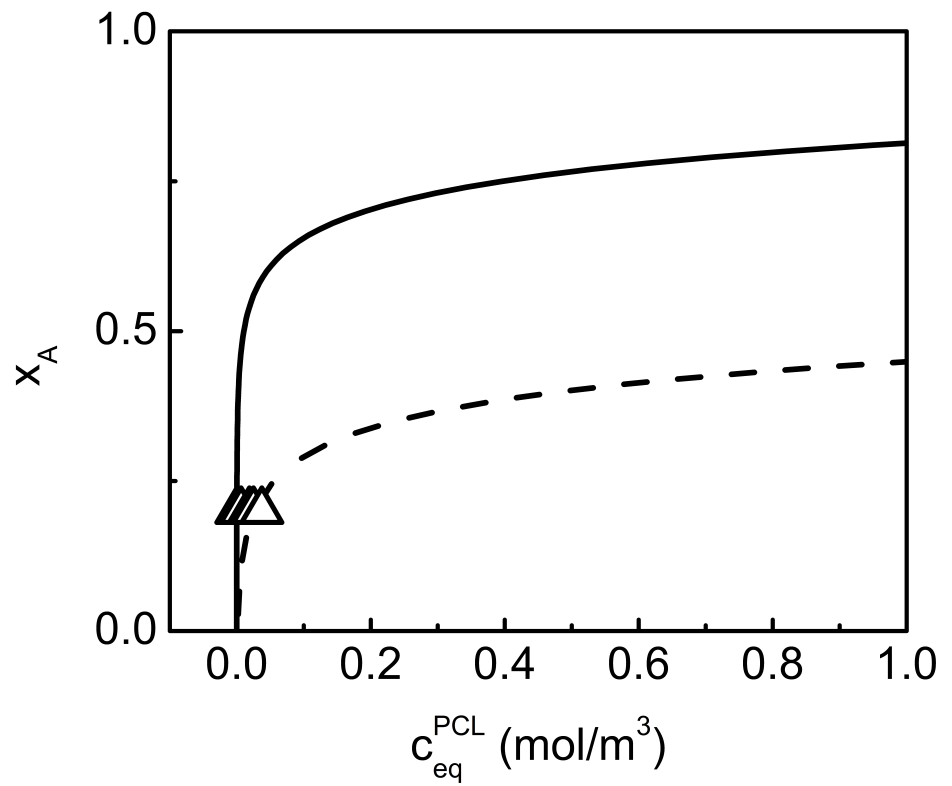

Figure 3: Solubility curve for PCL-80000 in acetone-water mixtures, reported as acetone molar fraction, $x_{A}$, versus the equilibrium concentration, $c_{\mathrm{eq}}^{\mathrm{PCL}}\left(x_{A}\right)$. Points above the curve correspond to stable mixture where PCL molecules do not aggregate, otherwise for the points below. The symbols (empty triangles) identify the six initial polymer concentrations, $c_{\text {out }}^{\mathrm{PCL}}$, for equal acetone and water flow rates, investigated in this work. The dashed curve corresponds to the supersaturation value equal to 200. The reason for choosing this latter value will be explained later on.

viscosity of the acetone-water mixture is instead calculated with the following empirical correlation:

$$
\mu=\exp \left[x_{A} \ln \left(\mu_{A}\right)+\left(1-x_{A}\right) \ln \left(\mu_{W}\right)\right],
$$

where $\mu_{A}$ and $\mu_{W}$ are the acetone and water viscosities, respectively, and $x_{A}$ is acetone molar fraction (evidently related to the acetone mass fraction). The Flory parameters, $k$ and $\nu$, for Eq. (4) and Eq. (5) are obtained by fitting MD simulations from our previous work (Di Pasquale et al. 2014) and depend on the acetone molar fraction as follows:

$$
\begin{gathered}
k=0.0064 \exp \left(-3.15 x_{A}\right), \\
\nu=0.30+0.45 x_{A}-0.15 x_{A}^{2} .
\end{gathered}
$$


It is interesting to notice that Eq. (11) returns for the exponent $\nu$ the theoretical values of $1 / 3$ and $3 / 5$ for bad (i.e. pure water) and good solvents (i.e. pure acetone), respectively.

As mentioned simulations were performed in a CIJM, which consists of two small (millimeter size) jets impinging at the center of a cylindrical chamber. The two jets, being confined in a small volume, after mixing exit from the bottom. Both top and bottom have conical ends. More specifically, the inlet diameter is equal to $1 \mathrm{~mm}$, whereas the outlet diameter is $2 \mathrm{~mm}$; the height and width correspond respectively to $9.52 \mathrm{~mm}$ and $4.76 \mathrm{~mm}$. More details concerning this particular mixer and its mixing features can be found in the literature (Icardi et al., 2011a b; Lince et al., 2009, 2011). By making use of its symmetry, one can simulate half of the geometry.

Ansys Fluent 15 was used in this work and DQMOM-IEM and QMOM were implemented via user-defined functions (UDF) and scalars (UDS). The computational grid consists of about 120.000 uniform hexahedral cells (for half of the actual geometry), with a refinement near the impinging plane and in the region around the two inlet flows. The pressure-velocity coupling has been done by using the SIMPLE algorithm, whereas the employed numerical scheme for spatial dicretization is the first-order upwind. In order to efficiently study turbulence inside the mixer the standard $k-\varepsilon$ model is employed with enhanced wall treatment near the wall, as this can be considered an adequate approach for this system (Gavi et al., 2007).

Outlet boundary conditions are set to zero normal gradients for all flow variables, except for pressure; in this way, outflow boundary values are not imposed but are calculated from the interior. As far as the boundary conditions for the moments of the CMD, $m^{(j)}$, are concerned they represents the initial PCL concentration (expressed in $\mathrm{mol} \mathrm{m} \mathrm{m}^{-3}$ ), as the PCL enter in the CIJM in a stable molecular solution, corresponding to a CMD centered on $n=1$ (i.e. clusters made up of only one PCL molecule). For this reason, this value is the same for the all four moments (i.e. $j=0, \ldots, 3$ ) calculated with the following equation:

$$
m^{(j)}=\frac{c_{\mathrm{in}}^{\mathrm{PCL}}}{M_{w} \rho_{\mathrm{A}}} \rho
$$

where $c_{\text {in }}^{\mathrm{PCL}}$ represents the initial PCL concentration expressed in $\mathrm{mg} \mathrm{mL}^{-1}$ and reported in Tab. 1. $M_{w}, \rho_{\mathrm{A}}$ and $\rho$ are respectively PCL molecular weight, acetone mixture and mixture density. Since PCL is dissolved in the acetone 
inlet stream, the boundary conditions related to the moments in the water inlet are equal to zero.

\section{Results and discussion}

Figure 4 reports the more significant contour plots in order to better understand the influence of fluid dynamics on the system. In particular we report the contour plots of the Fauvre-averaged velocity magnitude, of the moment of the CMD of order zero, $\langle m\rangle^{(0)}$ and of the mean radius of gyration, $\left\langle R_{g}\right\rangle$ of the polymer nanoparticles. It is worth stressing that the Fauvreaveraged mean radius of gyration, $\left\langle R_{g}\right\rangle$, is the mean radius of gyration of a population of nanoparticles, each characterized by its own individual radius of gyration, indicated as $\llbracket R_{g}{ }^{2}(n) \rrbracket$ in Eq. (5). These contour plots are reported in the symmetry plane of the CIJM for increasing flow rates of the two inlet streams of water and acetone. As it can be seen, the two inlet jets collide in an impinging plane, confined in the central part of the mixing chamber. This impinging plane is where most of the kinetic energy is produced and dissipated and is where mixing takes place. It is also interesting to notice that the impinging plane is slightly shifted towards the acetone inlet due to the difference of density between acetone and water. Closer observation of Fig. 4 reveals that the flow is laminar in the two inlets, as evident from the parabolic velocity profile, and is instead turbulent in other regions. It is also interesting to observe that some small stagnant zones are generated near the walls. This leads to a higher residence time of the fluid in these regions that, as we will see, influences directly the FNP process.

Concerning the turbulent kinetic energy, $k$, and the turbulence dissipation rate, $\varepsilon$, by increasing the inlet flow rates the characteristic values of both $k$ and $\varepsilon$ increase, indicating faster and faster mixing. The turbulence dissipation rate is particularly important as it determines the rate with which mixing at the molecular level, or micromixing, occurs (accounted for by DQMOMIEM). In other words the turbulent dissipation rate dictates the rate with which acetone (and PCL) and water molecules enter into contact, dictating therefore also the rate with which the FNP process occurs. The turbulent dissipation rate is also important because it defines the rate of aggregation due to turbulent fluctuations, as clearly evidenced by Eq. (6). In general, the effect of increasing $\varepsilon$ is twofold: on one hand it improves micromixing, leading to the formation of smaller nanoparticles, but on the other hand it increments aggregation, leading to their subsequent aggregation. Its final effect 


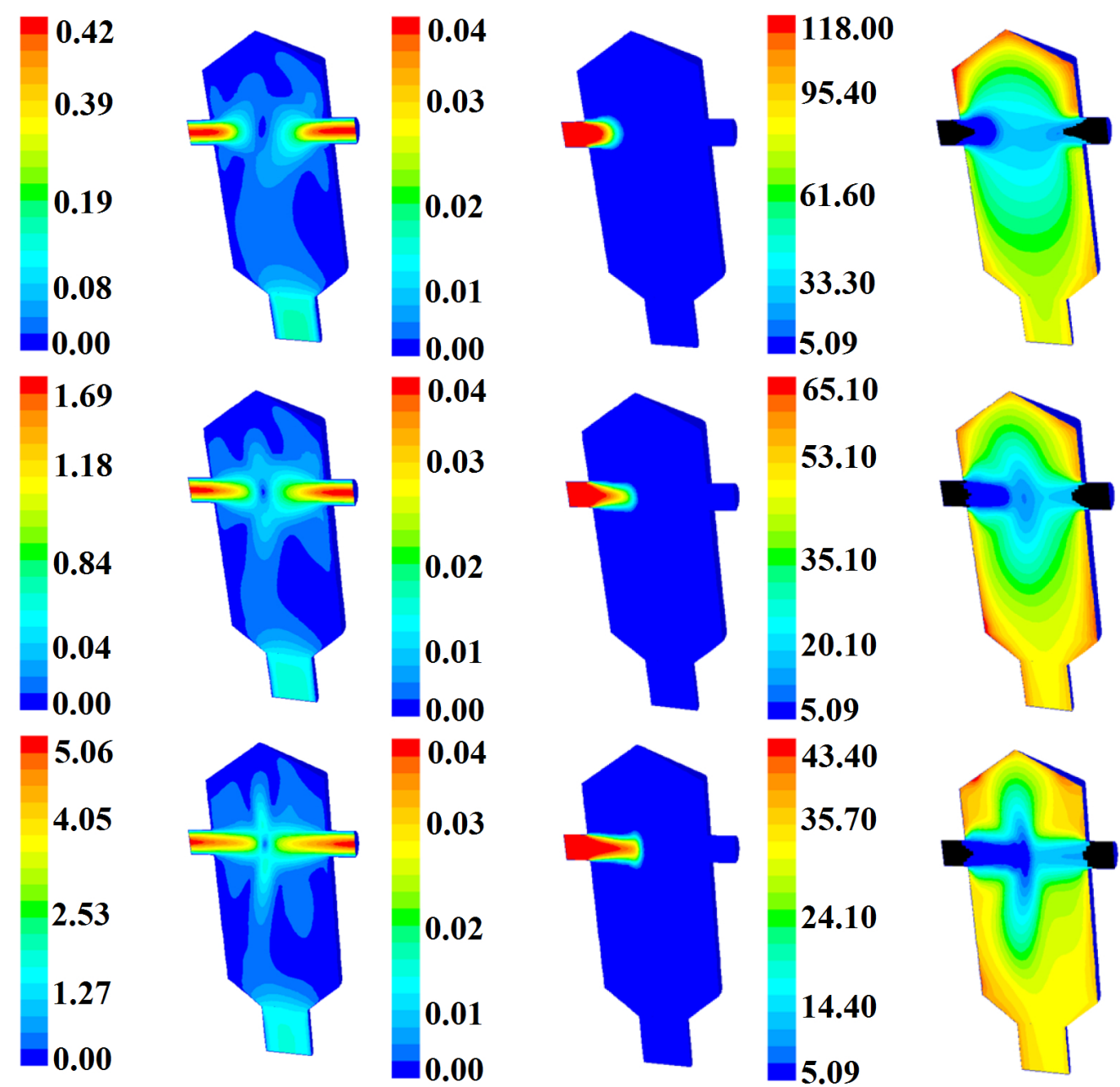

Figure 4: Contour plots for magnitude of the velocity vector $\left(\mathrm{m} \mathrm{s}^{-1}\right.$, left column), the 0thorder moment (center) and mean radius of gyration (nm, right column) on the symmetry plane of the CIJM for an initial PCL-14000 concentration of $0.5 \mathrm{mg} \mathrm{mL}^{-1}$ and for, from up to bottom, inlet flow rates of 10,40 and $120 \mathrm{~mL} \mathrm{~min}^{-1}$. 
of the final size of the aggregates is therefore difficult to predict a priori.

Figure 4 reports also the contour plots on the symmetry plane of the CIJM of the 0-th order moments of the CMD at different flow rates. Results are reported here only for PCL-14000 and for an initial PCL concentration in the acetone stream of $0.5 \mathrm{mg} \mathrm{mL} \mathrm{m}^{-1}$, as for the other molecular weight and for the other PCL initial concentrations very similar trends are observed. As already mentioned, $m^{(0)}$ represents the total nanoparticle number density. When PCL molecules enter the CIJM through the acetone inlet, they are in a stable molecular solution, and therefore each PCL molecule can be thought of as an independent nanoparticle/cluster characterized by a dimensionless mass, or aggregation number, of one (i.e. $n=1$ ). As the acetone stream mixes with the water stream the PCL equilibrium concentration is overcome, supersaturation is built up, and aggregation triggered, as depicted in Fig. 2 and 3. As soon as aggregation takes place, the original clusters $(n=1)$ aggregate forming larger nanoparticles/clusters, increasing $n$ and further reducing the total nanoparticle number density. For instance, if four molecules aggregate into one single nanoparticle/cluster, the total number density is reduced from four to one.

The contour plots of the Fauvre-averaged mean radius of gyration, $\left\langle R_{g}\right\rangle$, on the symmetry plane of the CIJM for different inlet flow rates is reported in right column of Fig. 4. As it can be noted, at the acetone inlet this quantity is very small (i.e. a few nanometers) and corresponds to the size of a single PCL-14000 molecule in pure acetone. As soon as water and acetone mix, molecules aggregate and the mean radius of gyration of the aggregates increases, reaching the final value at the outlet. It is interesting to observe that the maximum values for the radius of gyration are reached near the wall, where, as highlighted by the analysis of the flow field, stagnant zones are detected. In these stagnant zones the fluid and the nanoparticles spend more time and have therefore more time to aggregate. It is also interesting to observe that the mean radius of gyration sensibly decreases with the increase of the inlet flow rate. This reduction is due to the fact that, by increasing the inlet flow rate, faster mixing between the two inlet streams is achieved, resulting in the formation of more nanoparticles, that then have less PCL molecules available for subsequent growth via further aggregation.

By reconstructing the CMD, $f(n)$, as a lognormal distribution from the tracked four moments (Marchisio and Fox, 2013) at the CIJM outlet, the results reported in Fig. 5 are obtained. The data are reported here for different inlet flow rates, but one single PCL-14000 concentration (i.e. $0.5 \mathrm{mg} \mathrm{mL}{ }^{-1}$ ), 


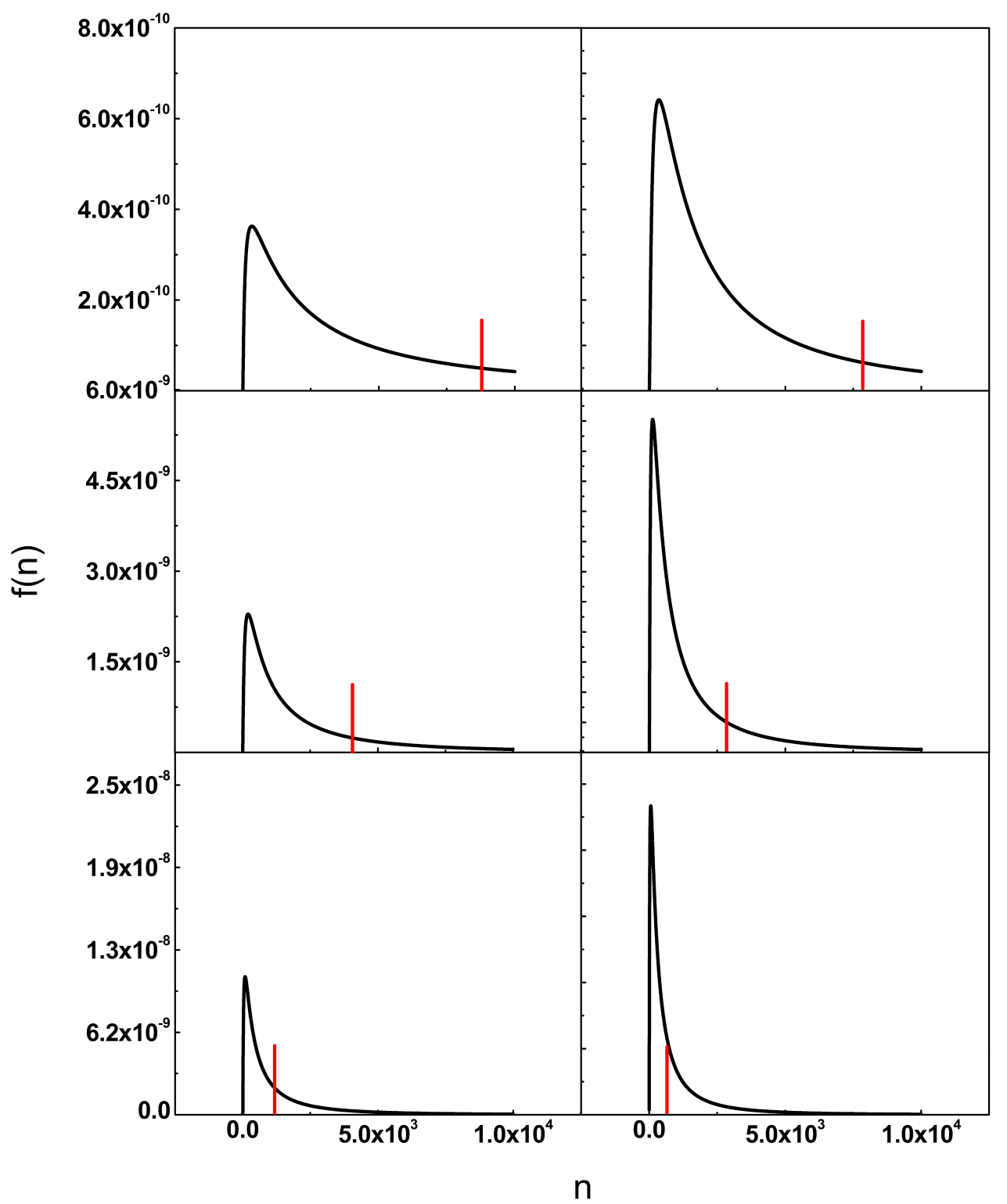

Figure 5: Reconstructed cluster mass distributions (CMD) at the CIJM outlet, $f(n)$, as a function of the number of molecules per nanoparticle, $n$, at PCL-14000 concentration of $0.5 \mathrm{mg} \mathrm{mL}-1$ and for, from left to right and top to bottom, inlet flow rates of 10, 20, 40, 60, 80 and $120 \mathrm{~mL} \mathrm{~min}{ }^{-1}$. The red vertical line indicates the mean value of $n$ of the distribution, namely $m^{(1)} / m^{(0)}$. 
and are plotted together with a line indicating the mean cluster mass (i.e. $\langle m\rangle^{(1)} /\langle m\rangle^{(0)}$ ) at the CIJM outlet. As already discussed, by increasing the inlet flow rates the CMD becomes narrower (i.e. the variance decreases), less skewed, whereas the mean cluster mass decreases. This confirms once again that manipulating the inlet flow rate allows to control the CMD. In particular, an increase in the inlet flow rates results in the formation of smaller nanoparticles with narrower CMD.

The mean radius of gyration at the reactor outlet can now be compared with experimental data collected in another work (Lince et al., 2008) by means of Dynamic Light Scattering (DLS). The comparison for the different PCL-14000 initial concentrations and for the different inlet flow rates investigated in this work is reported in Fig. 6. In general acceptable agreement between experiments and simulations (with Brownian and turbulent aggregation) is observed at all initial PCL-14000 concentrations, but the agreement improves as the concentration increases and becomes very good for concentrations equal to or higher than $2.5 \mathrm{mg} \mathrm{mL}^{-1}$. This corresponds to initial supersaturation (I.S.) greater than about 200.

For these simulations predictions are evaluated by considering the effect of Brownian aggregation only and of both Brownian and turbulent aggregation. The comparison of predictions in the two cases reveals that at low initial PCL-14000 concentrations the effect of turbulent motions is negligible on nanoparticle aggregation. This is probably due to the fact that at low initial PCL-14000 concentrations the nanoparticle size obtained from Brownian aggregation is too small to have some effect on turbulent fluctuations, as the turbulent aggregation kernel strongly depends on the cluster size. Under the operating conditions investigated in this work, the local turbulence intensities are such that turbulent aggregation is relevant only for cluster larger than $200 \mathrm{~nm}$.

As it can be seen, generally both experimental data and model predictions indicate a decrease of the nanoparticle mean radius of gyration with the inlet flow rate, as already observed and explained. The only exception is at the highest initial polymer concentration, where after a first decrease the mean radius of gyration increases at large inlet flow rates. This behavior, observed also in the experiments, is due again to the strong dependency of the aggregation kernel on the cluster size. Only at this high initial polymer concentration the nanoparticles become so large to be extremely sensitive to turbulent fluctuations. At this initial polymer concentration a first increase of the inlet flow rate results in the formation of more clusters, that then tend 


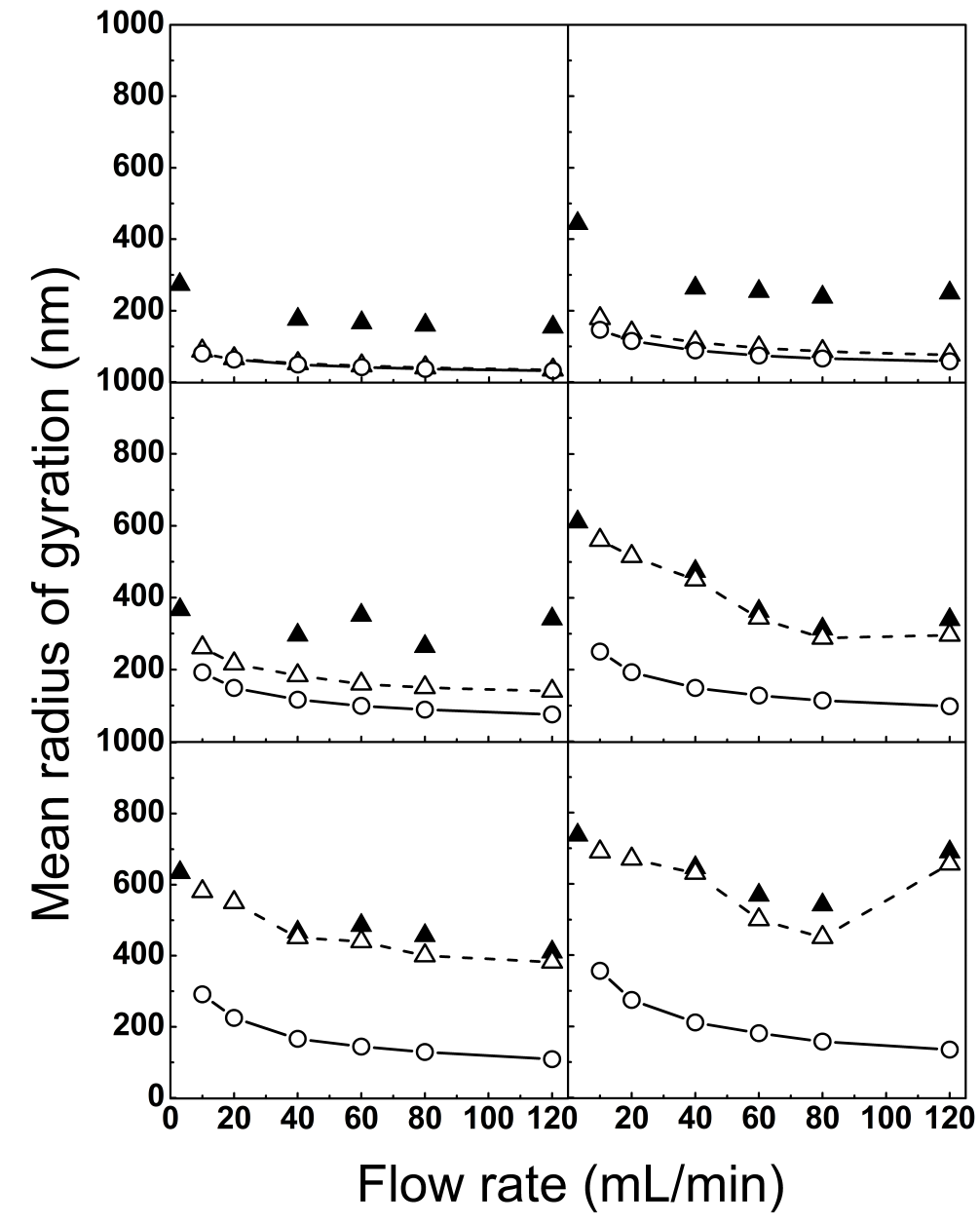

Figure 6: Mean radius of gyration of the nanopaticles exiting the CIJM versus the inlet flow rate as measured in experiments (black triangles) and as predicted by the model with only Brownian aggregation (continuous line) and Brownian and turbulent aggregation (dashed line) for PCL-14000 initial concentrations of, from left to right and top to bottom, of 0.5, $2.5,5.0,10.0,15.0$ and $25.0 \mathrm{mg} \mathrm{mL}^{-1}$. 
to grow smaller, but a further increase of the flow rate, results in an increase of the turbulent dissipation rate and of the resulting turbulent aggregation rate. This behavior is well reproduced by our model, confirming its validity.

The same trend is observed in Fig. 7 where model predictions are compared with experiments for PCL-80000. In this case model predictions due to both Brownian and turbulent aggregation are reported. The similarity of the trends confirms the generality of the model that can be therefore applied for both molecular weights. Also in this case predictions at low concentrations result in poorer agreement, when compared with higher concentrations. In this case, the agreement starts being acceptable for an initial PCL concentration of $6.0 \mathrm{mg} \mathrm{mL}^{-1}$, corresponding also in this case to an initial supersaturation larger than 200 .

Results seems to confirm that there exists a concentration range under which nanoparticle formation in FNP is controlled by Brownian aggregation and therefore can be described as a purely aggregative process. This happens for initial superaturation values greater than 200, whereas for initial supersaturation values smaller than 200 (for both the molecular weight considered in this work) the purely aggregative model does not result in good agreement, suggesting that a nucleative mechanism might control the process. Therefore, it is reasonable to infer that the spinodal line (i.e. the border line between the nucleation and the pure aggregation zones) could be placed at this value of initial supersaturation, as shown in Fig. 2 and 3.

\section{Conclusions}

In this work the FNP of PCL polymer molecules in acetone-water mixtures is studied by means of a multiscale modeling approach. The process is conducted inside a CIJM. The aim of the work is to develop a fully-predictive model capable of describing this phenomenon. The model developed has a general applicability and can therefore be applied also to other soft systems. The main novelty of this work lies on the use of a multiscale approach, capable of describing FNP at three scales: molecular-scale, nanoparticle-scale and macro-scale. It represents therefore an improvement with respect to other similar works (Tatek and Pefferkorn, 2004; Wang and Larson, 2015; Yuan et al., 2010) and it is consistent with other research efforts conducted in this area (Cheng et al., 2010; Li et al., 2006; Rajagopalan, 2001; Yan and Xie, 2013). The three scales communicate among each other, as suggested by other authors (Capretto et al., 2011; Rajagopalan, 2001), and are mod- 




Figure 7: Mean radius of gyration of the cluster exiting the CIJM versus the inlet flow rate as measured in experiments (black triangles) and as predicted by the model with only Brownian aggregation (continuous line) and Brownian and turbulent aggregation (dashed line) for PCL-80000 initial concentrations of, from left to right and top to bottom, of 0.02, $0.2,1.0,3.0,4.0$ and $6.0 \mathrm{mg} / \mathrm{mL}$. 
eled respectively with MD, Smoluchowski PBE and CFD, without resorting to other mesoscale techniques (Li et al., 2006, Yan and Xie, 2013). Another important novelty introduced in this work is the description of FNP as Brownian aggregation (i.e. free coupling mechanism, no energy barrier to overcome) coupled with turbulent flow models inside the CFD code, allowing the identification of kinetically-stable states, that would not be identified with other equilibrium models. Particularly important is the capability of the model to predict a change in the mean radius of gyration of the molecular clusters/nanoparticles of hundreds of nanometers by changing the inlet flow rates (i.e. turbulence of the system) of the water and acetone streams in the CIJM, without changing the chemical recipe. This latter aspect is particularly important when addressing the scale-up of the process to industrial scales or when investigating strategies to transfer the process to other continuous devices.

In order to assess the validity of the model, several operating conditions are evaluated in terms of initial PCL concentrations in the acetone inlet stream and in terms of inlet flow rate values. Moreover, two molecular weights for PCL are considered: 14000 and $80000 \mathrm{~g} \mathrm{~mol}^{-1}$. Simulation results are validated against experiments, resulting in good agreement, especially at high polymer concentrations. The agreement deteriorates as the polymer concentration is decreased, indicating that, in line with the corresponding theory, at low polymer concentrations and therefore low driving force for FNP, the role of nucleation starts being important. This allows moreover to assess the range of validity of the Brownian aggregation assumption. Future work is necessary in order to investigate other polymers, experimentally investigated in our previous work, and of the effect of other solvents.

\section{Acknowledgements}

The important contribution of Prof. Antonello Barresi is gratefully acknowledged, as well as the HPC@Polito Academic Computing Center.

\section{References}

Andersson, B., Andersson, R., Hakansson, L., Mortensen, M., Sudiyo, R., van Wachem, B., 2012. Computational fluid dynamics for engineering. Cambridge University Press. 
Baldyga, J., Orciuch, W., 2001. Some hydrodynamic aspects of precipitation. Powder Technol. 121, 9-19.

Bockstaller, M. R., Mickiewicz, R. A., Thomas, E., 2005. Block copolymer nanocomposites: Perspectives for tailored functional materials. Adv. Mater. 17, 1331-1349.

Capretto, L., Carugo, D., Cheng, W., Hill, M., Zhang, X., 2011. Continuousflow production of polymeric micelles in microreactors: Experimental and computational analysis. J. Colloid Interface Sci. 357, 243-251.

Celasco, E., Valente, I., Marchisio, D. L., Barresi, A. A., 2014. Dynamic light scattering and xray photoelectron spectroscopy characterization of pegylated polymer nanocarriers: Internal structure and surface properties. Langmuir 30, 8326-8335.

Cheng, J., Vigil, R., Fox, R., 2010. A competitive aggregation model for flash nanoprecipitation. J. Colloid Interface Sci. 351 (2), 330-342.

Cohen, S. I. A., Vendruscolo, M., , Dobson, C. M., , Knowles, T. P. J., 2011a. Nucleated polymerisation in the presence of pre-formed seed filaments. J. Chem. Phys. 135, 065107.

Cohen, S. I. A., Vendruscolo, M., , Dobson, C. M., , Knowles, T. P. J., 2011 b. Nucleated polymerisation in the presence of pre-formed seed filaments. Int. J. Mol. 12, 5844-5852.

Cohen, S. I. A., Vendruscolo, M., Dobson, C. M., , Knowles, T. P. J., 2011c. Proliferation of amyloid- $\beta 42$ aggregates occurs through a secondary nucleation mechanism. J. Chem. Phys. 135, 065106.

Cohen, S. I. A., Vendruscolo, M., Welland, M. E., Dobson, C. M., Terentjev, E. M., Knowles, T. P. J., 2011d. Nucleated polymerization with secondary pathways. i. time evolution of the principal moments. J. Chem. Phys. 135, 065105.

Collins, S. R., Douglass, A., Vale, R. D., Weissman, J. S., 2004. Mechanism of prion propagation: Amyloid growth occurs by monomer addition. PLoS Biol. 2, e321. 
Di Pasquale, N., Marchisio, D. L., Barresi, A. A., 2012. Model validation for precipitation in solvent-displacement processes. Chem. Eng. Sci. 84, 671-683.

Di Pasquale, N., Marchisio, D. L., Barresi, A. A., Carbone, P., 2014. Solvent structuring and its effect on the polymer structure and processability: the case of water-acetone poly- $\varepsilon$-caprolactone mixtures. J. Phys. Chem. B 118, $13258-13267$.

Di Pasquale, N., Marchisio, D. L., Carbone, P., Barresi, A. A., 2013. Identification of nucleation rate parameters with md and validation of the cfd model for polymer particle precipitation. Chem. Eng. Res. and Des. 91, 2275-2290.

Ferrone, F. A., Hofrichter, J., Eaton, W. A., 1985. Kinetics of sickle hemoglobin polymerization. ii. a double nucleation mechanism. J. Mol. Biol. 183, 611-631.

Flory, P., 1953. Principles of Polymer Chemistry. Cornell Univ. Press.

Fox, R., 2003. Computational models for turbulent reacting flows. Cambridge University Press.

Garcia, G. A., Cohen, S. I. A., Dobson, C. M., Knowles, T. P. J., 2014. Nucleation-conversion-polymerization reactions of biological macromolecules with prenucleation cluster. Phys. Rew. E 89, 032712.

Gavi, E., Rivautella, L., Marchisio, D. L., Vanni, M., Barresi, A. A., Baldi, G., 2007. Cfd modelling of nano-particle precipitation in confined impinging jet reactor. Chem. Eng. Sci. 85, 753-744.

Icardi, M., Gavi, E., Marchisio, D., Barresi, A., Olsen, M., Fox, R., Lakehal, D., 2011a. Investigation of the flow field in a three-dimensional confined impinging jets reactor by means of micropiv and dns. Chem. Eng. J. 166 (1), 294-305.

Icardi, M., Gavi, E., Marchisio, D., Olsen, M., Fox, R., Lakehal, D., 2011 b. Validation of les predictions for turbulent flow in a confined impinging jets reactor. App. Math. Mod. 35 (4), 1591-1602. 
Johnson, B. K., Prud'homme, R. K., 2003a. Chemical processing and micromixing in confined impinging jets. AIChE J. 49, 2264-2282.

Johnson, B. K., Prud'homme, R. K., 2003b. Flash nano-precipitation of organic actives and block copolymers using a confined impinging jets mixer. Aust. J. Chem 56, 1021-1024.

Jones, R. A. L., 2002. Soft condensed matter. Oxford Univ. Press.

Knowles, T. P. J., Waudby, C. A., Devlin, G. L., Cohen, S. I. A., Aguzzi, A., Vendruscolo, M., Terentjev, E. M., Welland, M. E., Dobson, C. M., 2009. An analytical solution to the kinetics of breakable filament assembly. Science 326, 1533-1537.

Li, Y. M., Xu, G. Y., Chen, Y. J., Luan, Y. X., Yuan, S. L., 2006. Computer simulations of surfactants and surfactant/polymer assemblies. Computational Materials Science 36, 386396.

Lince, F., Marchisio, D., Barresi, A., 2009. Smart mixers and reactors for the production of pharmaceutical nanoparticles: Proof of concept. Chem. Eng. Res. and Des. 87 (4), 543-549.

Lince, F., Marchisio, D., Barresi, A., 2011. A comparative study for nanoparticle production with passive mixers via solvent-displacement: Use of cfd models for optimization and design. Chem. Eng. Proc. 50 (4), 356-368.

Lince, F., Marchisio, D. L., Barresi, A. A., 2008. Strategies to control the particle size distribution of poly- $\varepsilon$-caprolactone nanoparticles for pharmaceutical applications. J. Colloid Interface Sci. 322, 505-515.

Maeda, H., 2001. Smancs and polymer-conjugated macromolecular drugs: advantages in cancer chemotherapy. Adv. Drug Delivery Rev. 46, 169-185.

Marchisio, D. L., Fox, R. O., 2013. Computational models for polydisperse particulate and multiphase flows. Cambridge University Press.

Marchisio, D. L., Vigil, R. D., Fox, R. O., 2003. Quadrature method of moments for aggregation-breakage processes. J. Colloid Int. Sci. 258, 322334.

Mura, F., Zaccone, A., 2016. Effects of shear flow on phase nucleation and crystallization. Physical Review E 93, 042803. 
Oosawa, F., Asakura, S., 1975. Thermodynamics of the Polymerization of Protein. Academic Press.

Oosawa, F., Kasai, M., 1962. A theory of linear and helical aggregations of macromolecules. J. Mol. Biol. 4, 10-21.

Rajagopalan, R., 2001. Simulations of self-assembling systemsl. Current Opinion in Colloid \& Interface Science 6, 357-365.

Rubinstein, M., Colby, R. H., 2003. Polymer Physics. Oxford Univ. Press.

Sun, H., Yang, X., 2014. Molecular simulation of self-assembly structure and interfacialinteraction for sdbs adsorption on graphene. Colloids and Surfaces A: Physicochem. Eng. Aspects 462, 82-89.

Tatek, Y., Pefferkorn, E., 2004. Clustercluster aggregation controlled by the number of intercluster connections: kinetics of aggregation and cluster mass frequency. J. Colloid Interface Sci. 278, 361-371.

Valente, I., Celasco, E., Marchisio, D. L., Barresi, A. A., 2012a. Nanoprecipitation in confined impinging jets mixers: Production, characterization and scale up of pegylated nanospheres and nanocapsules for pharmaceutical use. Chem. Eng. Sci. 77, 217-227.

Valente, I., Stella, B., Marchisio, D. L., Dosio, F., Barresi, A. A., 2012b. Production of pegylated nanocapsules through solvent-displacement in confined impinging jets mixers. J. Pharm. Sci. 101, 2490-2501.

Wang, S., Larson, R. G., 2015. Coarse-grained molecular dynamics simulation of self-assembly and surface adsorption of ionic surfactants using an implicit water model. Langmuir 31, 1262-1271.

Who, C., Jim, T., Gan, Z., Zhao, Y., Wang, S., 2000. A heterogeneous catalytic kinetics for enzymatic biodegradation of poly( $\varepsilon$-caprolactone) nanoparticles in aqueous solution. Polymer 41, 3593-3597.

Yan, L. T., Xie, X. M., 2013. Computational modeling and simulation of nanoparticle self-assembly in polymeric systems: Structures, properties and external field effects. Progress in Polymer Science 38, 369-405. 
Yuan, S. M., Yan, H., Lv, K., Liu, C. B., Yuan, S. L., 2010. Surface behavior of a model surfactant: A theoretical simulation study. J. Colloid Interface Sci. 348, 159-166.

Zhou, H. X., Ferrone, F. A., 1990. Theoretical description of the spatial dependence of sickle hemoglobin polymerization. Biophys. J. 58, 695-703. 